\title{
THE USE OF SIRE'S AND DAM'S RECORDS IN ANIMAL SELECTION
}

\author{
S. S. Y. YOUNG \\ Division of Animal Genetics, C.S.I.R.O., McMaster Laboratory, \\ Glebe, Sydney, N.S.W.
}

Received 22.xi.6o

\section{INTRODUCTION}

THE accuracy of estimation of an animal's breeding value can be increased by using its relatives' records as well as its own, in various combinations. Lush (1947) first derived optimal weighting coefficients for selection based on individual record and family average, and concluded that selection on such a combined score was never less efficient than selection on the individual's record only. Various systems of selection combining information on the individual and its, sibs and/or progeny have since been investigated by Lerner (I950), Osborne (1957 $a, b$ ) and Jardine (1958), while selection based on records of the individual and its ancestors has been discussed by Le Roy (1958) and Skjervold and Odegard (1959).

In farm animals, records of the dam or sire are useful when early selection is desirable or when additional accuracy is required. Dams' records are, of course, particularly useful in selection for sex-limited characters; for example, Robertson (1959) recently introduced an approximate method of pedigree evaluation with particular reference to dairy cattle. No investigation appears to have been made, however, of the method of combining the records of ancestors with those of contemporaries and/or progeny. Combinations of these types are useful when the records of dam and/or sire are readily available and when the number of offspring and sibs is small. In the present work the use of dam's and sire's records in some combinations is discussed, and explicit formulæ are presented for various types of combined selection involving records of dam and/or sire.

\section{THEORY \\ (i) Background}

The breeding value $(G)$ of an animal may be estimated from the performance of some or all of the following: its dam $(D)$, its sire $(F)$, its own phenotype $(I)$, the mean of its paternal half-sibs $(\bar{S})$, and the mean of its offspring $(\bar{O})$. In the present work combined selection using the information of $D$ and/or $F$ in various combinations with $I, S$ and $O$ (for example the combinations of $D$ and $I$, or $D, F$ and $\bar{O}$, etc.) are investigated, other combinations not involving $D$ and $F$ having been discussed in the papers cited earlier. However, some 
combinations such as that of $D, F, I, \bar{S}$ and $\bar{O}$, have not been investigated, as the computation becomes very tedious and the additional gain in accuracy by adding $D$ and/or $F$ to $I, \bar{S}$ and $\tilde{O}$ is probably small.

Selection based on records of full-sibs or maternal half-sibs has not been considered, as it is not usually practicable in farm animals such as cattle or sheep, with which pedigree records are most likely to be useful. However, the various combinations involving half-sibs can be adapted for full-sib selection by appropriate change of constants.

The prediction equation for $G$ when $D$ and $F$ are used in conjunction with other information is in the usual multiple regression form; for example, when these are used in combination with $\bar{S}$ we have an index,

$$
(\hat{G}-\bar{G})=b_{1}(D-\bar{P})+b_{2}(F-\bar{P})+b_{3}(\bar{S}-\bar{P})
$$

where $\bar{G}$ and $\bar{P}$ are the genetic and phenotypic means of the population respectively, and the $b_{i}$ 's are the appropriate weightings to be given to the different items of information. When only one item of information is available the above equation, of course, reduces to the simple regression form.

It has been shown by Hazel (1943) that when an index is used in selection the genetic gain $(\Delta G)$ can be estimated by

$$
\Delta G=i R_{G I} \sigma_{G}
$$

where $i$ is the selection differential in standard units, $R_{G I}$ the multiple correlation coefficient between the index and the breeding value and $\sigma_{G}^{2}$ is the additive genetic variance. It is also known that the variance of the estimate

$$
V(\Delta \hat{G}-\Delta G)=\left(\mathrm{I}-R_{G I}^{2}\right) \sigma_{G}^{2}
$$

$R_{G I}$ therefore is useful in the estimation of genetic gain as well as in the calculation of confidence limits for $\Delta G$. In the following pages formulæ for $b_{i}$ and $R_{G I}$ for various combinations are given, but before presenting the formulæ several pertinent points need to be mentioned.

(i) In the development of the formulæ it is assumed that the individual and its relatives have the same population mean. When the means are different, correction of records is necessary before the formulæ can be applied.

(ii) It is assumed that the animals under consideration are not inbred.

(iii) In the calculation of $\bar{S}$, the individual's own record is not included. When the individual record is available, it will be used as additional independent information on $G$.

In calculating the partial regression coefficients, it is necessary to write down the theoretical correlation coefficients between records of 
different relatives. Such coefficients have been derived and are presented in matrix form as:

\begin{tabular}{|c|c|c|c|c|c|c|c|c|}
\hline & $G$ & $D$ & $D$ & $F$ & $F$ & $I$ & $\bar{S}$ & $\bar{o}$ \\
\hline$G$ & I & $\frac{1}{2} h$ & $\frac{1}{2} h \sqrt{K}$ & $\frac{1}{2} h$ & $\frac{1}{2} h \sqrt{\mathcal{J}}$ & $h$ & $\frac{1}{4} h \sqrt{\mathcal{N}}$ & $\frac{1}{2} h \sqrt{Q}$ \\
\hline$D$ & & I & $\ldots$ & 0 & 0 & $\frac{1}{2} h^{2}$ & 0 & $\frac{1}{4} h^{2} \sqrt{Q}$ \\
\hline$D$ & & & I & o & o & $\frac{1}{2} h^{2} \sqrt{K}$ & o & $\frac{1}{4} h^{2} \sqrt{K Q}$ \\
\hline$F$ & & & & I & $\ldots$ & $\frac{1}{2} h^{2}$ & $\frac{1}{2} h^{2} \sqrt{ } \overline{\mathcal{N}}$ & $\frac{1}{4} h^{2} \sqrt{Q}$ \\
\hline $\bar{F}$ & & & & & I & $\frac{1}{2} h^{2} \sqrt{\mathcal{J}}$ & $\frac{1}{2} h^{2} \sqrt{\overline{\mathcal{J N}}}$ & $\frac{1}{4} h^{2} \sqrt{\mathcal{J} Q}$ \\
\hline I & & & & & & I & $\frac{1}{4} h^{2} \sqrt{\mathcal{N}}$ & $\frac{1}{2} h^{2} \sqrt{Q}$ \\
\hline $\bar{S}$ & & & & & & & I & $\frac{1}{8} h^{2} \sqrt{Q \mathcal{N}}$ \\
\hline$\overline{0}$ & & & & & & & & I \\
\hline
\end{tabular}

where $D=$ mean of $k$ records on the dam,

$F=$ mean of $j$ records on the sire,

$h^{2}=$ value of heritability,

$\kappa=\frac{k}{\mathrm{I}+(k-\mathrm{I}) T}, T$ being the value of repeatability,

$\mathcal{J}=\frac{j}{\mathrm{I}+(j-\mathrm{I}) \mathcal{T}}$,

$\mathcal{N}=\frac{n}{\mathrm{I}+(n-\mathrm{I}) t}, n$ being the number of half sibs, and $t$ the for half sibs),

$Q=\frac{q}{\mathrm{I}+(q-\mathrm{I}) t}, q$ being the number of progeny.

It is seen that when $k=j=n=q=\mathrm{I}$ then $K=\mathcal{J}=\mathcal{N}=Q=\mathrm{I}$, so that formulæ derived for means of any type of record can readily be reduced to cases when only a single record of each kind is available.

With these coefficients it is possible, by standard multiple regression analysis, to calculate the appropriate weights to be given to each item of information in a given combination, as well as the multiple correlation coefficient between the resulting index and the animal's breeding value. The formulæ are presented in the order of:

(a) The means used in prediction; the combination of the mean record of the dam and the record of the individual, for example, is written as $D+I$.

(b) The corresponding partial regression coefficients $\left(b_{i}\right)$.

(c) The multiple correlation coefficient $(R)$.

Only combinations involving means of relatives are given; for example, instead of showing 4 sets of combinations $D+F, \bar{D}+F$, 
$D+F, D+F$ only formula involving $D+F$ are listed. If a combination such as $D+F$ is required, then suitable formula can easily be derived from those concerning $D+F$ by making $K=\mathcal{J}=\mathrm{r}$.

(ii) Weighting and multiple correlation coefficients for different combinations of records

(i) Combinations in which records of the mean of dam $(D)$ or sire $(F)$ can be interchanged. (When sire mean is used, change $K$ to $\mathcal{F}$ ).

(1) (a) $D$ (or $F$ )

(b) $b=\frac{1}{2} h^{2} K$

(c) $r=\frac{1}{2} h \sqrt{K}$.

(2) (a) $D$ (or $F)+I$

(b) $b_{1}=\frac{2 h^{2} K\left(1-h^{2}\right)}{4-h^{4} K}$

$b_{2}=\frac{h^{2}\left(4-h^{2} K\right)}{4-h^{4} K}$

(c) $R=h \sqrt{\frac{4+K\left(\mathrm{I}-2 h^{2}\right)}{4-h^{4} K}}$.

(3) (a) $D$ (or $F)+O$

(b) $b_{1}=\frac{2 h^{2} K\left(4-h^{2} Q\right)}{16-h^{4} K Q}$

$$
b_{2}=\frac{2 h^{2} Q\left(4-h^{2} K\right)}{16-h^{4} K Q}
$$

(c) $R=h \sqrt{\frac{2\left[2 K+Q\left(2-h^{2} K\right)\right]}{\mathrm{I} 6-h^{4} K Q}}$.

(4) (a) $D$ (or $F)+I+D$

(b) $b_{1}=\frac{2 h^{2} K\left(\mathrm{I}-h^{2}\right)\left(4-h^{2} Q\right)}{\mathrm{I} 6-h^{4}\left[4(K+Q)+K Q\left(\mathrm{I}-2 h^{2}\right)\right]}$

$b_{2}=\frac{h^{2}\left(4-h^{2} K\right)\left(4-h^{2} Q\right)}{\mathrm{I} 6-h^{4}\left[4(K+Q)+K Q\left(\mathrm{I}-2 h^{2}\right)\right]}$

$b_{3}=\frac{2 h^{2} Q\left(\mathrm{I}-h^{2}\right)\left(4-h^{2} K\right)}{\mathrm{I} 6-h^{4}\left[4(K+Q)+K Q\left(\mathrm{I}-2 h^{2}\right)\right]}$

(c) $R=h \sqrt{\frac{\mathrm{I} 6+4(K+Q)\left(\mathrm{I}-2 h^{2}\right)-h^{2} K Q\left(2-3 h^{2}\right)}{\mathrm{I} 6-h^{4}\left[4(K+Q)+K Q\left(\mathrm{I}-2 h^{2}\right)\right]}}$. 
(ii) Combinations involving records of the dam.

(I) (a) $D+S$

(b) $b_{1}=\frac{1}{2} h^{2} K$

$b_{2}=\frac{1}{4} h_{N}^{2}$

(c) $R=\frac{1}{4} h \sqrt{4 K+\mathcal{N}}$.

(2) (a) $D+I+S$

(b) $b_{1}=\frac{8 h^{2} K\left(\mathrm{I}-h^{2}\right)}{16-h^{4}(4 K+\mathcal{N})}$

$$
\begin{aligned}
& b_{2}=\frac{h^{2}\left[16-h^{2}\left({ }^{2} K+\mathcal{N}\right)\right]}{16-h^{4}(4 K+\mathcal{N})} \\
& b_{3}=\frac{4 h^{2} \mathcal{N}\left(1-h^{2}\right)}{16-h^{4}\left({ }^{4} K+\mathcal{N}\right)}
\end{aligned}
$$

(c) $R=h \sqrt{\frac{16+(4 K+\mathcal{N})\left(\mathrm{I}-2 h^{2}\right)}{\mathrm{I} 6-h^{4}(4 K+\mathcal{N})}}$.

(3) (a) $\bar{D}+\bar{S}+\bar{O}$

(b) $b_{1}=\frac{8 h^{2} K\left(4-h^{2} Q\right)}{64-h^{4} Q(4 K+\mathcal{N})}$

$$
\begin{aligned}
& b_{2}=\frac{4 h^{2} \mathcal{N}\left(4-h^{2} Q\right)}{64-h^{4} Q(4 K+\mathcal{N})} \\
& b_{3}=\frac{2 h^{2} Q\left[\mathrm{I} 6-h^{2}(4 K+\mathcal{N})\right]}{64-h^{4} Q(4 K+\mathcal{N})}
\end{aligned}
$$

(c) $R=h \sqrt{\frac{2\left[8 Q+(4 K+\mathcal{N})\left(2-h^{2} Q\right)\right]}{64-h^{4} Q(4 K+\mathcal{N})}}$.

(iii) Combinations involving records of the sire.

(1) (a) $F+\bar{S}$

(b) $b_{1}=\frac{h^{2} \mathcal{F}\left(4-h^{2} \mathcal{N}\right)}{2\left(4-h^{4} \mathcal{G N}\right)}$

$$
b_{2}=\frac{h^{2} \mathcal{N}\left(\mathrm{I}-h^{2} \mathcal{J}\right)}{4-h^{4} \mathcal{J N}}
$$

(c) $R=\frac{1}{2} h \sqrt{\frac{4 \mathcal{A}+\mathcal{N}\left(\mathrm{I}-h^{2} \mathcal{F}\right)}{4-h^{4} \mathcal{J N}}}$. 
(2) (a) $F+I+\bar{S}$

(b) $b_{1}=\frac{2 h^{2} \mathcal{J}\left(\mathrm{I}-h^{2}\right)\left(4-h^{2} \mathcal{N}\right)}{\mathrm{I} 6-h^{4}\left[4 \mathcal{J}(\mathrm{I}+\mathcal{N})+\mathcal{N}\left(\mathrm{I}-2 h^{2} \mathcal{J}\right)\right]}$

$$
\begin{aligned}
& b_{2}=\frac{h^{2}\left\{\mathrm{I} 6-h^{2}\left[\mathcal{N}+2 \mathcal{J}\left(2+h^{2} \mathcal{N}\right)\right]\right\}}{\mathrm{I} 6-h^{4}\left[4 \mathcal{J}(\mathrm{I}+\mathcal{N})+\mathcal{N}\left(\mathrm{I}-2 h^{2} \mathcal{J}\right)\right]} \\
& b_{3}=\frac{4 h^{2} \mathcal{N}\left(\mathrm{I}-h^{2}\right)\left(\mathrm{I}-h^{2} \mathcal{J}\right)}{\mathrm{I} 6-h^{4}\left[4 \mathcal{J}(\mathrm{I}+\mathcal{N})+\mathcal{N}\left(\mathrm{I}-2 h^{2} \mathcal{J}\right)\right]}
\end{aligned}
$$

(c) $R=h \sqrt{\frac{2\left(8-h^{2} \mathcal{J N}\right)+\left(\mathrm{I}-2 h^{2}\right)(4 \mathcal{J}+\mathcal{N})}{\mathrm{I} 6-h^{4}\left[4 \mathcal{J}(\mathrm{I}+\mathcal{N})+\mathcal{N}\left(\mathrm{I}-2 h^{2} \mathcal{J}\right)\right]}}$.

(3) (a) $F+\bar{S}+\bar{O}$

(b) $b_{1}=\frac{2 h^{2} \mathcal{J}\left(4-h^{2} \mathcal{N}\right)\left(4-h^{2} Q\right)}{64-h^{4}\left[4 \mathcal{J}(4 \mathcal{N}+Q)+Q \mathcal{N}\left(\mathrm{I}-2 h^{2} \mathcal{J}\right)\right]}$

$$
b_{2}=\frac{4 h^{2} \mathcal{N}\left(4-h^{2} Q\right)\left(\mathrm{I}-h^{2} \mathcal{J}\right)}{64-h^{4}\left[4 \mathcal{J}(4 \mathcal{N}+Q)+Q \mathcal{N}\left(\mathrm{I}-2 h^{2} \mathcal{J}\right)\right]}
$$$$
b_{3}=\frac{2 h^{2} Q\left[4\left(4-h^{2} \mathcal{J}\right)-h^{2} \mathcal{N}\left(\mathrm{I}+2 h^{2} \mathcal{J}\right)\right]}{64-h^{4}\left[4 \mathcal{J}(4 \mathcal{N}+Q)+Q \mathcal{N}\left(\mathrm{I}-2 h^{2} \mathcal{J}\right)\right]}
$$

(c) $R=h \sqrt{\frac{2\left[8(\mathcal{J}+Q)-4 h^{2} \mathcal{J}(\mathcal{N}+Q)+\mathcal{N}\left(2-h^{2} Q\right)\right]}{64-h^{4}\left[4 \mathcal{J}(4 \mathcal{N}+Q)+Q \mathcal{N}\left(\mathrm{I}-2 h^{2} \mathcal{J}\right)\right]}}$.

(iv) Combinations involving records of both dam and sire.

(I) (a) $D+F$

(b) $b_{1}=\frac{1}{2} h^{2} K$

$b_{2}=\frac{1}{2} h^{2} J$

(c) $R=\frac{1}{2} h \sqrt{K+\mathcal{J}}$.

(2) (a) $D+F+I$

(b) $b_{1}=\frac{2 h^{2} K\left(\mathrm{I}-h^{2}\right)}{4-h^{4}(K+\mathcal{J})}$

$$
b_{2}=\frac{2 h^{2} \mathcal{J}\left(\mathrm{I}-h^{2}\right)}{4-h^{4}(K+\mathcal{J})}
$$

$b_{3}=\frac{h^{2}\left[4-h^{2}(K+\mathcal{J})\right]}{4-h^{4}(K+\mathcal{J})}$

(c) $R=h \sqrt{\frac{4+(K+\mathcal{J})\left(\mathrm{I}-2 h^{2}\right)}{4-h^{4}(K+\mathcal{J})}}$.

(3) (a) $\bar{D}+\bar{F}+\bar{S}$

(b) $b_{1}=\frac{1}{2} h^{2} K$

$$
\begin{aligned}
& b_{2}=\frac{h^{2} \mathcal{J}\left(4-h^{2} \mathcal{N}\right)}{2\left(4-h^{4} \mathcal{J N}\right)} \\
& b_{3}=\frac{h^{2} \mathcal{N}\left(\mathrm{I}-h^{2} \mathcal{J}\right)}{4-h^{4} \mathcal{J N}}
\end{aligned}
$$


(c) $R=\frac{1}{2} h \sqrt{\frac{\mathcal{N}+4(K+\mathcal{J})-h^{2} \mathcal{J N}\left(2+h^{2} K\right)}{\left(4-h^{4} \mathcal{J} \mathcal{N}\right)}}$.

(4) (a) $\bar{D}+\bar{F}+\bar{O}$

(b) $b_{1}=\frac{2 h^{2} K\left(4-h^{2} Q\right)}{\mathrm{I} 6-h^{4} Q(K+\mathcal{J})}$

$$
\begin{aligned}
b_{2} & =\frac{2 h^{2} \mathcal{J}\left(4-h^{2} Q\right)}{\mathrm{I} 6-h^{4} Q(K+\mathcal{J})} \\
b_{3} & =\frac{2 h^{2} Q\left[4-h^{2}(K+\mathcal{J})\right]}{\mathrm{I} 6-h^{4} Q(K+\mathcal{J})} \\
\text { (c) } R & =h \sqrt{\frac{2\left[\left(2-h^{2} Q\right)(K+\mathcal{J})+2 Q\right]}{\mathrm{I} 6-h^{4} \ell(K+\mathcal{J})} .}
\end{aligned}
$$

(5) (a) $D+F+I+\bar{S}$

(b) $b_{1}=\frac{2 h^{2} K\left(4-h^{4} \mathcal{J N}\right)\left(\mathrm{I}-h^{2}\right)}{\mathrm{I} 6-h^{4}\left[4(\mathcal{K}+\mathcal{J})+\mathcal{N}(\mathrm{I}+4 \mathcal{J})-h^{2} \mathcal{J} \mathcal{N}\left(h^{2} K+2\right)\right]}$

$$
\begin{aligned}
b_{2} & =\frac{2 h^{2} \mathcal{J}\left(4-h^{2} \mathcal{N}\right)\left(\mathrm{I}-h^{2}\right)}{\mathrm{I} 6-h^{4}\left[4(K+\mathcal{J})+\mathcal{N}(\mathrm{I}+4 \mathcal{J})-h^{2} \mathcal{J N}\left(h^{2} K+2\right)\right]} \\
b_{3} & =\frac{h^{2}\left\{\mathrm{I} 6-h^{2}\left[\mathcal{N}+4(K+\mathcal{J})+h^{2} \mathcal{J N}\left(2-h^{2} K\right)\right]\right\}}{\mathrm{I} 6-h^{4}\left[4(K+\mathcal{J})+\mathcal{N}(\mathrm{I}+4 \mathcal{J})-h^{2} \mathcal{J N}\left(h^{2} K+2\right)\right]} \\
b_{4} & =\frac{4 h^{2} \mathcal{N}\left(\mathrm{I}-h^{2} \mathcal{J}\right)\left(\mathrm{I}-h^{2}\right)}{\mathrm{I} 6-h^{4}\left[4(K+\mathcal{J})+\mathcal{N}(\mathrm{I}+4 \mathcal{J})-h^{2} \mathcal{J N}\left(h^{2} K+2\right)\right]} \\
\text { (c) } R & =h \sqrt{\frac{2\left(8-h^{2} \mathcal{J N}\right)+\left[4(K+\mathcal{J})+\mathcal{N}\left(\mathrm{I}-h^{4} \mathcal{J} K\right)\right]\left(\mathrm{I}-2 h^{2}\right)}{\mathrm{I} 6-h^{4}\left[4(\mathcal{K}+\mathcal{J})+\mathcal{N}(\mathrm{I}+4 \mathcal{J})-h^{2} \mathcal{J N}\left(h^{2} K+2\right)\right]}} .
\end{aligned}
$$

(6) (a) $D+F+I+\bar{O}$

(b) $b_{1}=\frac{2 h^{2} K\left(4-h^{2} Q\right)\left(\mathrm{I}-h^{2}\right)}{\mathrm{I} 6-h^{4}\left[4(K+\mathcal{J}+Q)+Q(K+\overline{\mathcal{J}})\left(\mathrm{I}-2 h^{2}\right)\right]}$

$$
\begin{aligned}
b_{2} & =\frac{2 h^{2} \mathcal{J}\left(4-h^{2} Q\right)\left(\mathrm{I}-h^{2}\right)}{\mathrm{I} 6-h^{4}\left[4(K+\mathcal{J}+Q)+Q(K+\mathcal{J})\left(\mathrm{I}-2 h^{2}\right)\right]} \\
b_{3} & =\frac{h^{2}\left\{\mathrm{I} 6-h^{2}\left[4(K+\mathcal{J}+Q)-h^{2} Q(K+\mathcal{J})\right]\right\}}{\mathrm{I} 6-h^{4}\left[4(K+\mathcal{J}+Q)+Q(K+\mathcal{J})\left(\mathrm{I}-2 h^{2}\right)\right]} \\
b_{4} & =\frac{2 h^{2} Q\left[4-h^{2}(K+\mathcal{J})\right]\left(\mathrm{I}-h^{2}\right)}{\mathrm{I} 6-h^{4}\left[4(K+\mathcal{J}+Q)+Q(K+\mathcal{J})\left(\mathrm{I}-2 h^{2}\right)\right]} \\
\text { (c) } R & =h \sqrt{\frac{\mathrm{I} 6+4\left(\mathrm{I}-2 h^{2}\right)(K+\mathcal{J}+Q)-h^{2} Q\left(2-3 h^{2}\right)(K+\mathcal{J})}{\mathrm{I} 6-h^{4}\left[4(K+\mathcal{J}+Q)+Q(K+\mathcal{J})\left(\mathrm{I}-2 h^{2}\right)\right]}} .
\end{aligned}
$$

\section{DISCUSSION}

It is usually worthwhile to use records such as $D$ or $F$ to increase the accuracy of selection when they are readily available, despite 
some additional computation. When such information has to be specially obtained then it is desirable to know the relative efficiency of different systems of combined selection.

A detailed examination of the relative efficiency of all the combinations presented here would be extremely lengthy, but a general discussion and some specific examples may be of interest.

The relative efficiency of any two systems depends on such factors as the number of different items of records used, the correlation between records of relatives and the breeding value of the animal and the values of heritability under consideration. The value of heritability for any given character can be regarded as given so that the efficiency of any system depends largely on the types of records used and the correlation between the records and the breeding value. The most useful types of records, of course, would be those highly correlated with $G$. The correlation between a record and $G$ is determined, apart from the genetic relationships between the record and $G$, by factors $K$, or $\mathcal{N}$ etc. (see matrix of correlation), large values of $K, \mathcal{N}$, etc. tending to increase the value of the correlation coefficients.

As is implicit in earlier discussions, values of $K, \mathcal{N}$, etc., are never less than I and it can be shown that their values increase with increasing $k, n$, etc. Since

$$
\frac{\partial K}{\partial k}=\frac{\mathrm{I}-T}{[\mathrm{I}+(k-\mathrm{I}) T]^{2}} \text { and } \frac{\partial \mathcal{N}}{\partial n}=\frac{\mathrm{I}-t}{[\mathrm{I}+(n-\mathbf{I}) t]^{2}} \text {, etc. }
$$

and because $(0 \leqq T, t \leqq I)$ the above differential coefficients are nonnegative. As $k$ or $n$ etc., increases we have

$$
\lim _{k \rightarrow \infty} K=\frac{\mathrm{I}}{T} \text {, and } \lim _{n \rightarrow \infty} \mathcal{N}=\frac{\mathrm{I}}{t} \text { etc., }
$$

so that $\left(\mathrm{I} \leqq \mathcal{J}, K<\frac{\mathrm{I}}{T}\right)$ and $\left(\mathrm{I} \leqq \mathcal{N}, Q<\frac{\mathrm{I}}{t}\right)$. These results are intuitively clear since the usefulness of family records increases with increasing family size and with decreasing heritability of the trait under selection (or repeatability in case of mean records of parents), so that $D$ and $F$ are most useful when $k$ and $j$ are large and when $n$ and $q$ are small.

As a first illustration, consider the relative efficiency of the individual's own record (mass selection) and the combination $D+F+I$. In such comparisons it is convenient to assume that the proportion of animals selected with either method is constant. The relative efficiency of any two systems, in terms of genetic gain, is then the ratio of the two correlation coefficients between the breeding value and the respective selection indices. In the present case the relative superiority of the system $D+F+I$ over mass selection is

$$
E_{1}=\sqrt{\frac{4+(K+\mathcal{J})\left(\mathrm{I}-2 h^{2}\right)}{4-h^{4}(K+\mathcal{J})}}
$$


Values of $E_{1}$ for (i) $k=j=\mathrm{I}$, (ii) $k=j=2$, (iii) $k=j=3$, (iv) $k=j=4$ and $T=\mathrm{I} \cdot 30 h^{2}$ have been calculated and are plotted in fig. 1. The superiority of combined selection is greatest when $k$ and

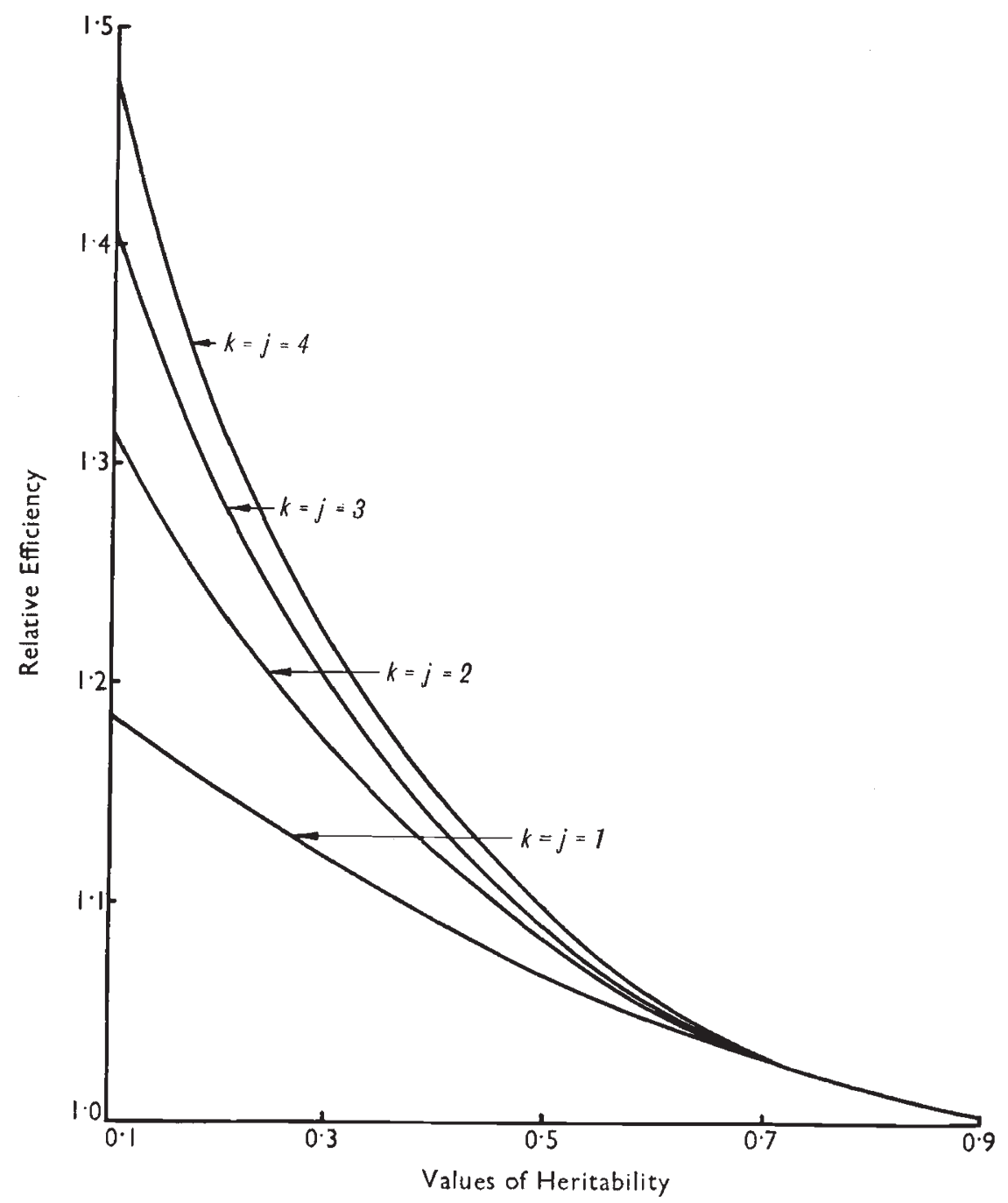

FIG. 1.-The relative efficiency of combined selection (using records of dam, sire and individual) and mass selection (using individual only).

Note : Relative efficiency $=\frac{\text { Gain by combined selection }}{\text { Gain by mass selection }}$,

$k=$ number of records on dam,

$j=$ number of records on sire.

Repeatability has been assumed 30 per cent. higher than heritability.

$j$ are large and $h^{2}$ low. When $h^{2}$ is high the gain by combined selection is small irrespective of the sizes of $k$ and $j$. But when $h^{2}$ is low (say, $0 \cdot 2$ ), even when $k=j=\mathrm{I}$, combined selection offers an advantage 
of I 5 per cent. and when $h^{2}=0 \cdot$ I combined selection is superior by I 8 per cent.

As another illustration consider a male selection programme for a sex-limited character; the relative efficiency of the index $\bar{D}+\bar{S}$ and progeny testing $(\bar{O})$ will be of interest in this case. The relative

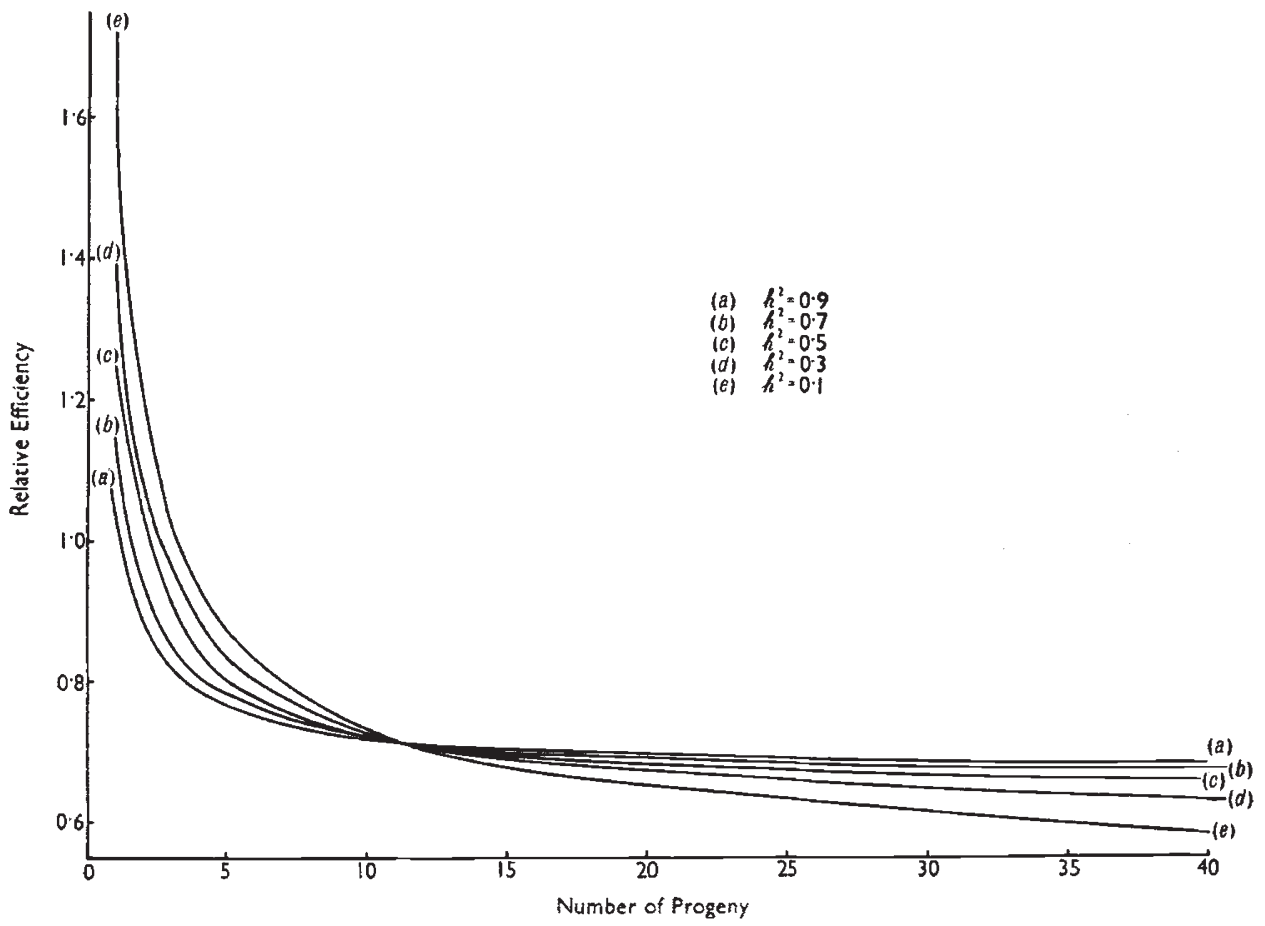

FIG. 2.- The relative efficiency of combined selection (using records of dam and halfsibs) and selection based on progeny mean.

Note : Relative efficiency $=\frac{\text { Gain by combined selection }}{\text { Gain by progeny testing }}$.

Repeatability has been assumed 30 per cent. higher than heritability.

efficiency, in terms of genetic gain per generation from the selected males, can be calculated as

$$
E_{2}=\frac{1}{2} \sqrt{\frac{4 K+\mathcal{N}}{Q}}
$$

where $E_{2}$ is a ratio of gain by using $D+\bar{S}$ to gain by progeny testing. Values of $E_{2}$ are plotted against $n$ in fig. 2 under the conditions of
(i) $n=q$,
(ii) $k=3$,
(iii) $T=\mathrm{I} \cdot 30 h^{2}$, with different values of $h^{2}$.

It should be noted that when the dam's records are ignored, half-sib testing is only 50 per cent. as efficient as progeny testing, in terms of 
gain per generation. In fig. 2, therefore, values of $E_{2}$ tend towards 0.5 as $n$ increases. When $n$ is small, $E_{2}$ can be greater than I, so that under our present conditions $D+\bar{S}$ is then more efficient. An interesting feature of fig. 2 is that the addition of $D$ in the index can increase the relative efficiency of sib-testing considerably even when $n$ is quite large. This is particularly so when $h^{2}$ is high. Thus when $n=35$, under the present set of conditions the addition of $D$ has contributed approximately 14 per cent. to the relative efficiency when $h^{2}=0.3$, 16 per cent. when $h^{2}=0.5$ and 18 per cent. when $h^{2}=0.7$.

Genetic gain per generation in the above comparison, however, is not a realistic criterion. As has been pointed out by many workers in this field (Dickerson and Hazel, 1944; Rendel and Robertson, I950, etc.) factors such as generation length, flock structure, and the relative costs etc. must also be considered. Another factor which is in favour of the $D+\bar{S}$ system is the fact that it may be possible to examine the records of dams and sibs of all the male animals under consideration, but it is almost impossible to progeny test them all. If this is the case the selection intensity for the $D+S$ system would be higher than that of progeny testing and it follows that the selection differential, in standard deviation units, (i) for $D+\bar{S}$ would also be higher. Hence the relative efficiency of $\bar{D}+\bar{S}$, in reality, would usually be higher than it is indicated by values of $E_{2}$. In such cases, the ratio of relative efficiency of the two systems may be calculated as $E_{2}^{\prime}=E_{2}\left(i_{1} / i_{2}\right)$, where $i_{1}$ and $i_{2}$ are the $i$ values for the systems $D+\bar{S}$ and $\delta$ respectively.

All the above comments are well known to animal breeders but it seems pertinent to point out the various important considerations associated with any family selection problems.

\section{SUMMARY}

The use of records of the dam and the sire in various combinations with the record of the individual, sibs average, and progeny average is investigated. Explicit formulæ for 16 different combinations are given. These sets of formulæ can be applied in selection when the relatives have different amounts of information. Systems of combined selection investigated in this work are useful when it is desirable to select animals early in life, or when additional accuracy in selection is required. Records of the dams in combination with other information are particularly useful in selection for sex-limited characters. The relative efficiency of some systems of combined selection are discussed as part of a general comment on the relative efficiencies of different systems of combined selection.

Acknowledgments.-Thanks are due to Miss Helen Newton Turner of the Division of Animal Genetics for her helpful comments, and to Miss Elaine Smith and Dr G. M. Tallis of the same Division for checking most of the formulæ presented in this work. 


\section{REFERENCES}

DiCKERSON, G. E., AND HAZEL, L. N. 1944. Effectiveness of selection on progeny performance as a supplement to early culling in livestock. 7. Agric. Res., 69, $459-476$.

HAZEL, L. N. 1943. The genetic basis for constructing selection indexes. Genetics, $28,476-490$.

JARDINE, R. 1958. Animal breeding and the estimation of genetic value. Heredity, I2, 499-5II.

LE ROY, H. L. I958. Die Abstammungsbewertung. Z. Tierz. ZuchtBiol., 71, 328-378. Lerner, I. M. 1950. Population Genetics and Animal Improvement. Cambridge Univ. Press.

LUSH, J. L. 1947. Family merit and individual merit as bases for selection. I. II. Amer. Nat., 81, 241-261, 362-379.

OSBORNE, R. I957a. The use of sire and dam family averages in increasing the efficiency of selective breeding under a hierarchical mating system. Heredity, II, 93-II6.

OSBORNE, R. 1957b. Family selection in poultry: the use of sire and dam family averages in choosing male parents. Proc. Roy. Soc. Edin., 66, 374-393.

RENDEL, J. M., AND ROBERTSON, A. 1950. Estimation of genetic gain in milk yield by selection in a closed herd of dairy cattle. 7. Genet., 50 , I-8.

ROBERTSON, A. 1959. A simple method of pedigree evaluation in dairy cattle. Anim. Prod. 1, 167-1 74.

SKJERVOLD, H., AND ODEGARD, A. K. 1959. Estimation of breeding value on the basis of the individual's own phenotype and ancestors' merits. Acta. agric. Scand., 9, 34 I-354. 